\title{
Ultra Low Temperature Freezer
}

National Cancer Institute

\section{Source}

National Cancer Institute. Ultra Low Temperature Freezer. NCI Thesaurus. Code C107385.

A type of freezer that can maintain temperatures between -80 and -86 degrees C. 